\title{
ANALYSIS OF THE RATIONAL KRYLOV SUBSPACE PROJECTION METHOD FOR LARGE-SCALE ALGEBRAIC RICCATI EQUATIONS *
}

\author{
V. SIMONCINI ${ }^{\dagger}$
}

\begin{abstract}
In the numerical solution of the algebraic Riccati equation $A^{*} X+X A-X B B^{*} X+$ $C^{*} C=0$, where $A$ is large, sparse and stable, and $B, C$ have low rank, projection methods have recently emerged as a possible alternative to the more established Newton-Kleinman iteration. In spite of convincing numerical experiments, a systematic matrix analysis of this class of methods is still lacking. We derive new relations for the approximate solution, the residual and the error matrices, giving new insights into the role of the matrix $A-B B^{*} X$ and of its approximations in the numerical procedure. The new results provide theoretical ground for recently proposed modifications of projection methods onto rational Krylov subspaces.
\end{abstract}

Key words. Riccati equation, rational Krylov, reduced order modelling

AMS subject classifications. 47J20, 65F30, 49M99, 49N35, $93 \mathrm{~B} 52$

1. Introduction. We consider the numerical solution of the algebraic Riccati equation

$$
A^{*} X+X A-X B B^{*} X+C^{*} C=0,
$$

where $A \in \mathbb{R}^{n \times n}$ is large and sparse, and $B \in \mathbb{R}^{n \times q}, C \in \mathbb{R}^{p \times n}$ with $q, p \ll n$; here and in the following $A^{*}$ denotes the complex conjugate of $A$. For $A$ stable 1 , the solution matrix $X$ of interest is the one that is symmetric positive semidefinite and such that $A-B B^{*} X$ remains stable. Equation (1.1) arises in many scientific and engineering applications that require controlling a dynamical system, and it has been deeply studied by applied algebraists and numerical mathematicians; we refer the reader to [29] for a thorough description of the problem and its many mathematical relations. In the recent book [11, the numerical treatment of this and related problems has been discussed, both in the small and large scale cases. In the large scale setting, with $n \gg 10^{3}$, a serious bottleneck is given by the fact that the possibly dense $n \times n$ matrix $X$ cannot be stored. Most numerical methods thus approximate $X$ by means of factored low-rank matrices, e.g., $X \approx Z Z^{*}$, so that only $Z$ needs to be stored. Different approaches have been explored to solve (1.1) under this constraint, and for quite some time a variant of the Newton method, the Newton-Kleinman iteration, has been the most popular approach [28, [16, [10, , 8]. Low-rank subspace iteration strategies have also been considered in the past few years, see, e.g., [1, 17, [31]. Other forms of datasparse approximations include multilevel [19] and hierarchical 20] methods, which rely on available structure in the data.

Projection-type methods also yield low rank approximations, however they had not been used for the Riccati equation until very recently. In fact, projection methods are extensively employed in the solution of algebraic linear systems and eigenvalue problems. In the past decade, specific choices of approximation spaces have shown that projection methods are particularly effective also for linear matrix equations such as the Lyapunov and Sylvester equations 38. Lately, the projection idea has been applied to the algebraic (quadratic) Riccati equation [26, [25], with surprisingly good

\footnotetext{
${ }^{*}$ Version of January 31, 2016.

${ }^{\dagger}$ Dipartimento di Matematica, Università di Bologna, Piazza di Porta S. Donato 5, I-40127 Bologna, Italy and IMATI-CNR, Pavia, Italy (valeria.simoncini@unibo.it).

${ }^{1} \mathrm{An} n \times n$ matrix is stable if all its eigenvalues are in the open left half complex plane.
} 
results, to the point that better performance is often observed than with Newtonbased procedures [40]. Moreover, ad-hoc parameter selections have been proposed to further enhance particularly effective approximation spaces [31. This strong numerical evidence however is lacking of any theoretical justification: the procedure is mainly based on its linear counterpart and therefore it seems to completely disregard the quadratic term $-X B B^{*} X$. Nonetheless, fast convergence to the sought after solution is usually observed.

The aim of this paper is to start an analysis that will lead to a better understanding of this class of methods. By looking at the computed quantities from different perspectives, we are able to give new insights into the role of the approximate solution $X_{k}$ in the various contexts where the Riccati equation is extensively studied. We start in section 3 with model order reduction of linear dynamical systems, where approximation by projection is a recognized important tool, and show that $X_{k}$ carries information on the optimal function value in the reduced control problem. In section 5 we deepen our knowledge of $X_{k}$ and the associated residual, which allows us to derive new expressions for the residual matrix and justify recently proposed enhancements of a popular space in model order reduction, that is the rational Krylov subspace. A key role in our discussion will be played by the residual matrix,

$$
R_{k}:=A^{*} X_{k}+X_{k} A-X_{k} B B^{*} X_{k}+C^{*} C .
$$

By simple algebra, it is customary to rewrite $R_{k}$ as

$$
R_{k}=\left(A^{*}-X_{k} B B^{*}\right) X_{k}+X_{k}\left(A-B B^{*} X_{k}\right)+C^{*} C+X_{k} B B^{*} X_{k},
$$

which highlights the occurrence of the matrix $A^{*}-X_{k} B B^{*}$. This matrix and its projected version will be ubiquitous in the paper, and are the true players in the approximation process. Finally, the connection between the approximation of the matrix equation and the invariant subspace setting is highlighted in section 6. While our interest was motivated by the good performance of rational Krylov methods, which are the main focus of section 5. many of the results in fact hold for more general projection methods. We believe that our analysis helps provide good ground to characterize projection methods as a natural and effective strategy for solving the Riccati equation.

The following notation and definitions will be used. For $X \in \mathbb{R}^{n \times n}, X \geq 0$ means that $X$ is symmetric and positive semidefinite, while $X>0$ means that is symmetric and positive definite. A stable matrix is a square matrix with all its eigenvalues in the open left-half complex plane. An $n \times n$ matrix $A$ is passive if its field of values, $\left\{z \in \mathbb{C}: z=\left(x^{*} A x\right) /\left(x^{*} x\right), 0 \neq x \in \mathbb{C}^{n}\right\}$, is all in the open left-half complex plane. $I_{n}$ denotes the identity matrix of size $n$, and the subscript will be avoided whenever clear from the context. A pair $(A, B)$ is controllable if the matrix $\left[B, A B, \ldots, A^{n-1} B\right]$ is full row rank, and $(C, A)$ is observable if $\left(A^{*}, C^{*}\right)$ is controllable. A pair $(A, B)$ is stabilizable if there exists a matrix $X$ such that $A-B B^{*} X$ is stable. The Euclidean norm $\|\cdot\|$ for vectors and its induced norm for matrices will be used, together with the Frobenius norm for matrices, defined as $\|A\|_{F}^{2}=\sum_{i, j}\left|a_{i, j}\right|^{2}$, where $A=\left(a_{i, j}\right)$.

2. Background on projection methods. Projection methods usually generate a sequence of nested approximation spaces, $\mathcal{K}_{k} \subseteq \mathcal{K}_{k+1}, k \geq 1$, where an approximate solution is determined. Let the columns of $V_{k} \in \mathbb{R}^{n \times d_{k}}$ span the space $\mathcal{K}_{k}$, where $d_{k}$ is the space dimension, with $d_{k} \leq d_{k+1}$. An approximation to $X$ in (1.1) is sought as $X_{k}=V_{k} Y_{k} V_{k}^{*} \approx X$, where $Y_{k}$ is determined by imposing some additional condition. 
A Galerkin method is characterized by an orthogonality condition of the residual to the given space, namely $R_{k} \perp \mathcal{K}_{k}$, where $R_{k}$ is as defined in (1.2); the orthogonality is with respect to the standard matrix inner product, so that the Galerkin condition reads

$$
V_{k}^{*} R_{k} V_{k}=0
$$

As the subspace grows, the residual is forced to belong to a smaller and smaller space. When $d_{k}=n$ then clearly it must be $R_{k}=0$ and a solution to (1.1) is determined, in exact arithmetic. The main goal is to determine a sufficiently good approximate solution $X_{k}$ for $d_{k} \ll n$. To obtain $Y_{k}$ we substitute $X_{k}$ into the expression for the residual matrix in (2.1):

$$
\begin{aligned}
V_{k}^{*}\left(A^{*} V_{k} Y_{k} V_{k}^{*}+V_{k} Y_{k} V_{k}^{*} A-V_{k} Y_{k} V_{k}^{*} B B^{*} V_{k} Y_{k} V_{k}^{*}+C^{*} C\right) V_{k} & =0 \\
V_{k}^{*} A^{*} V_{k} Y_{k}+Y_{k} V_{k}^{*} A V_{k}-Y_{k} V_{k}^{*} B B^{*} V_{k} Y_{k}+V_{k}^{*} C^{*} C V_{k} & =0,
\end{aligned}
$$

where we used that $V_{k}^{*} V_{k}=I_{d_{k}}$. Setting $T_{k}=V_{k}^{*} A V_{k}, B_{k}=V_{k}^{*} B$ and $C_{k}^{*}=V_{k}^{*} C^{*}$ we see that $Y_{k}$ can be obtained by solving the reduced Riccati equation

$$
T_{k}^{*} Y_{k}+Y_{k} T_{k}-Y_{k} B_{k} B_{k}^{*} Y_{k}+C_{k}^{*} C_{k}=0 .
$$

Under the assumption that $A$ is passive, $T_{k}$ is stable, therefore (2.2) admits a unique stabilizing positive semidefinite solution $Y_{k}$, which is then used for constructing $X_{k}$.

The effectiveness of the whole procedure depends on the choice of $\mathcal{K}_{k}$. The approximation spaces explored in the (quite recent) literature are all based on block Krylov subspaces generated with $A$ or with rational functions of $A$ and starting term $C^{*}$ [26, 25], 240]. In section [5 we will analyze the case of the block rational Krylov subspace, while the results of the next two sections hold for any approximation space.

3. Order reduction of dynamical systems by projection. The Riccati equation is tightly connected with the time-invariant linear system

$$
\left\{\begin{array}{l}
\dot{x}(t)=A x(t)+B u(t), \quad x(0)=x_{0} \\
y(t)=C x(t)
\end{array}\right.
$$

where $u(t)$ and $x(t)$ are the control (or input) and state vectors, while $y(t)$ is the output vector; $x_{0}$ is the initial state. We note that $x(t)$ also depends on both $x_{0}$ and $u(t)$, but this will not be explicitly reported in the notation. Let us introduce the following quadratic cost functiona

$$
\mathcal{J}\left(u, x_{0}\right)=\int_{0}^{\infty}\left(x(t)^{*} C^{*} C x(t)+u(t)^{*} u(t)\right) d t .
$$

The Riccati equation matrix $X$ is used in the solution of the following linear-quadratic regulator problem:

$$
\inf _{u} \mathcal{J}\left(u, x_{0}\right)
$$

which consists in finding an optimal control function $u_{*}(t)$ associated with the system (3.1), at which the function $\mathcal{J}$ attains its infimum. The following well known result

\footnotetext{
${ }^{2}$ Here we consider a simplified version to make an immediate connection with the Riccati equation stated in 1.1).
} 
connects the optimal cost problem with the solution of the algebraic Riccati equation (1.1); see, e.g., the relevant part of [29, Theorem 16.3.3] in our notation.

Theorem 3.1. Let the pair $(A, B)$ be stabilizable and $(C, A)$ observable. Then there is a unique solution $X \geq 0$ of (1.1). Moreover,

i) For each $x_{0}$ there is a unique optimal control, and it is given by $u_{*}(t)=$ $-B^{*} X \exp \left(\left(A-B B^{*} X\right) t\right) x_{0}$ for $t \geq 0$;

ii) $J\left(u_{*}, x_{0}\right)=x_{0}^{*} X x_{0}$ for all $x_{0} \in \mathbb{C}^{n}$.

The optimal control function $u_{*}(t)$ in the theorem above is in fact determined as $u_{*}(t)=-B^{*} X x(t)$, giving rise to the closed-loop dynamical system

$$
\dot{x}(t)=\left(A-B B^{*} X\right) x(t), \quad x(0)=x_{0},
$$

whose solution is $x(t)=\exp \left(\left(A-B B^{*} X\right) t\right) x_{0}$ for $t \geq 0$ [24].

A reduced order model aims at representing the given large dynamical system by means of a significantly smaller one. This can be done by projecting data onto a significantly smaller space. A popular strategy in this class is to use the Rational Krylov subspace to reduce the coefficient matrices by projecting them onto an appropriate vector space [3]. The solutions of the reduced system can effectively approximate the original state and control in case the space trajectories do not occupy the whole state space. In practice, this means that the original model can be well represented by far fewer degrees of freedom [2].

A quantity of interest to the control community that is used to monitor the quality of the reduced system is the transfer function, for which a large literature is available; see, e.g., [3], [9], [18, [37] and their references. Here we focus on the reduction process, and show that the subspace projection allows one to determine the optimal control of the reduced dynamical system. Let the $d_{k} \ll n$ orthonormal columns of $V_{k} \in \mathbb{R}^{n \times d_{k}}$ span the computed subspace, and, as in the previous section, let $T_{k}=V_{k}^{*} A V_{k}, B_{k}=$ $V_{k}^{*} B, C_{k}^{*}=V_{k}^{*} C^{*}$. Then we can define the reduced order system

$$
\left\{\begin{array}{l}
\dot{\hat{x}}(t)=T_{k} \widehat{x}(t)+B_{k} \widehat{u}(t), \quad \widehat{x}(0)=V_{k}^{*} x_{0} \\
\widehat{y}(t)=C_{k} \widehat{x}(t)
\end{array}\right.
$$

Clearly, as $d_{k} \rightarrow n$ the reduced system approaches the original one. For smaller $d_{k}$, the quantity $x_{k}(t)=V_{k} \widehat{x}(t)$ is an approximate state of the original system.

Corollary 3.2. The solution matrix $Y_{k}$ of (2.2) is the unique non-negative solution that gives the feedback optimal control $\widehat{u}_{*}(t), t \geq 0$, for the system (3.2).

Proof. Let

$$
\widehat{\mathcal{J}}_{k}\left(\widehat{u}, \widehat{x}_{0}\right)=\int_{0}^{\infty}\left(\widehat{x}(t)^{*} C_{k}^{*} C_{k} \widehat{x}(t)+\widehat{u}(t)^{*} \widehat{u}(t)\right) d t .
$$

be the cost functional associated with (3.2). By applying Theorem 3.1 an optimal control for the reduced system is $\widehat{u}_{*}(t)=-B_{k}^{*} Y_{k} \exp \left(\left(T_{k}-B_{k} B_{k}^{*} Y_{k}\right) t\right) \widehat{x}_{0}$, where $Y_{k}$ solves the reduced Riccati equation

$$
T_{k}^{*} Y+Y T_{k}-Y B_{k} B_{k}^{*} Y+C_{k}^{*} C_{k}=0,
$$

with the reduced state $\widehat{x}(t)=\exp \left(\left(T_{k}-B_{k} B_{k}^{*} Y_{k}\right) t\right) \widehat{x}_{0}$. Equation (3.3) is precisely the Riccati equation obtained by Galerkin projection of the original large scale matrix equation (1.1) onto the given subspace. 
Theorem 3.1(ii) implies

$$
\widehat{\mathcal{J}}_{k}\left(\widehat{u}_{*}, \widehat{x}_{0}\right)=\widehat{x}_{0}^{*} Y_{k} \widehat{x}_{0}=x_{0}^{*} V_{k} Y_{k} V_{k}^{*} x_{0}=x_{0}^{*} X_{k} x_{0} .
$$

Therefore, if $X_{k} \rightarrow X$ as $d_{k} \rightarrow \infty$, the optimal value of the reduced functional yields an estimate to the minimum functional cost via the approximate solution $X_{k}=V_{k} Y_{k} V_{k}^{*}$ to the large Riccati equation.

An approximate control function $u_{k}(t)$ for the unreduced functional $\mathcal{J}\left(u_{k}, x_{0}\right)$ is obtained directly using the approximation $X_{k}$, bypassing the reduced functional $\widehat{\mathcal{J}}$. Indeed, if we assume that the approximate Riccati solution $X_{k}$ is stabilizing, we can write

$$
u_{k}(t)=-B^{*} X_{k} x_{k}(t), \quad \text { with } \quad x_{k}(t)=\exp \left(\left(A-B B^{*} X_{k}\right) t\right) x_{0} .
$$

Substituting $X_{k}=V_{k} Y_{k} V_{k}^{*}$ we get $u_{k}(t)=-\left(B^{*} V_{k}\right) Y_{k} V_{k}^{*} \exp \left(\left(A-B\left(B^{*} V_{k}\right) Y_{k} V_{k}^{*}\right) t\right) x_{0}$. The question then arises as of whether $u_{k}$ and $\widehat{u}_{*}$ are related. Comparing this expression with that of $\widehat{u}_{*}(t)$, we see that they are close to each other as soon as

$$
\exp \left(\left(V_{k}^{*}\left(A-B B X_{k}\right) V_{k} t\right) V_{k}^{*} \approx V_{k}^{*} \exp \left(\left(A-B B^{*} X_{k}\right) t\right) .\right.
$$

Using the expansion of $\exp (z)$ in terms of power series and taking transpose conjugations, this approximation can be written as

$$
\left(A^{*}-X_{k} B B^{*}\right)^{\ell} V_{k} \approx V_{k}\left(V_{k}^{*}\left(A^{*}-X_{k} B B^{*}\right) V_{k}\right)^{\ell}, \quad \text { for any } \ell \in \mathbb{N} .
$$

This approximation becomes an equality as soon as range $\left(V_{k}\right)$ is an invariant subspace of $A^{*}-X_{k} B B^{*}$. In general, however, the columns of $V_{k}$ do not span an invariant subspace, therefore this connection is not sufficient to connect the two control functions. The following proposition does provide a relation between the optimal reduced cost functional value with the value of the original functional at $u_{k}$.

Proposition 3.3. Assume that $A-B B^{*} X_{k}$ is stable and that $u_{k}$ is defined as in (3.4). With the previous notation it holds

$$
\left|\mathcal{J}\left(u_{k}, x_{0}\right)-\widehat{\mathcal{J}}_{k}\left(\widehat{u}_{*}, \widehat{x}_{0}\right)\right| \leq \frac{\left\|R_{k}\right\|}{2 \alpha} x_{0}^{*} x_{0},
$$

where $\alpha>0$ is such that $\left\|e^{\left(A-B B^{*} X_{k}\right)^{*} t}\right\| \leq e^{-\alpha t}$ for all $t \geq 0$.

Proof. Using (1.3), let us write the Riccati residual equation as

$$
\left(A-B B X_{k}^{*}\right)^{*} X_{k}+X_{k}\left(A-B B X_{k}\right)+X_{k} B B^{*} X_{k}+C^{*} C-R_{k}=0 .
$$

Then

$$
\begin{aligned}
\mathcal{J}\left(u_{k}, x_{0}\right) & =\int_{0}^{\infty}\left(u_{k}^{*} u_{k}+x_{k}^{*} C^{*} C x_{k}\right) d t \\
& =\int_{0}^{\infty} x_{0}^{*} e^{\left(A-B B^{*} X_{k}\right)^{*} t}\left(X_{k} B B^{*} X_{k}+C^{*} C\right) e^{\left(A-B B^{*} X_{k}\right) t} x_{0} d t \\
& =x_{0}^{*} X_{k} x_{0}+\int_{0}^{\infty} x_{0}^{*} e^{\left(A-B B^{*} X_{k}\right)^{*} t} R_{k} e^{\left(A-B B^{*} X_{k}\right) t} x_{0} d t .
\end{aligned}
$$

From $x_{0}^{*} X_{k} x_{0}=\widehat{J}_{k}\left(\widehat{u}_{*}, \widehat{x}_{0}\right)$ and $\left|\int_{0}^{\infty} x_{0}^{*} e^{\left(A-B B^{*} X_{k}\right)^{*} t} R_{k} e^{\left(A-B B^{*} X_{k}\right) t} x_{0} d t\right| \leq \frac{\left\|R_{k}\right\|}{2 \alpha} x_{0}^{*} x_{0}$ the result follows. 
This theorem establishes a linear relation between the matrix equation residual norm and the distance between the optimal value of the reduced functional and the value of the approximate unreduced functional. As the residual norm goes to zero, the two functional values coalesce, and this may occur for $d_{k} \ll n$, that is with a projection space of much smaller dimension than the original one.

We conclude with a remark about the type of approximation space used. In model order reduction, usually different projection spaces are used from the left and from the right, so as to expand both in terms of $C^{*}$ and $B$. The connection between this approach and the reduction of the (symmetric) Riccati equation deserves future analysis.

4. Control stability properties of the subspace projection approximation. By using the residual equation, norm estimates for the error $X-X_{k}$ can be derived by using classical perturbations results. In this section we recall these classical estimates, which can have a different flavor in our setting, where the perturbations are not very small in general. Nonetheless, these results enable us to state that for $d_{k}$ large enough the approximate solution $X_{k}$ is rigorously equipped with all the nice stabilizability properties of the exact solution. Moreover, they can be used to track the progress in the approximation as the approximation space grows.

Unlike the linear equation case, a small residual norm does not necessarily imply a small error, since the Riccati equation has more than one solution. Therefore, in general an assumption is needed about the closeness of the approximate solution to the sought after one, to be able to derive information on the error norm from the residual norm.

Let $X$ be an exact stabilizing solution, $E_{k}=X-X_{k}$ the error and $R_{k}=A^{*} X_{k}+$ $X_{k} A-X_{k} B B^{*} X_{k}+C^{*} C$ the residual. Subtract this residual equation from (1.1). Then by adding and subtracting $X B B^{*} X_{k}$ and $E_{k} B B^{*} X_{k}$ in sequence, we obtain

$$
\left(A^{*}-X B B^{*}\right) E_{k}+E_{k}\left(A-B B^{*} X\right)+E_{k} B B^{*} E_{k}+R_{k}=0 .
$$

We observe in passing that the second order term in $E_{k}$ becomes negligeable for $\left\|E_{k}\right\| \ll 1$. From this Riccati equation for the error, under certain conditions a bound on the error can be obtained. To this end we recall the definition of the closed-loop Lyapunov operator

$$
\Omega_{X}(Z):=\left(A-B B^{*} X\right)^{*} Z+Z\left(A-B B^{*} X\right),
$$

and observe that if $H$ is the matrix solving $\left(A-B B^{*} X\right)^{*} H+H\left(A-B B^{*} X\right)=-I$, then $\|H\|=\left\|\Omega_{X}^{-1}\right\|=\max _{Z \neq 0}\left(\left\|\Omega_{X}^{-1}(Z)\right\| /\|Z\|\right)$; see [27, Lemma 2]. Note that $\left\|\Omega_{X}^{-1}\right\|$ is the reciprocal of the sep operator for the given matrix [41. An interesting interpretation of $\left\|\Omega_{X}^{-1}\right\|$ in terms of the damping of the closed-loop dynamical system is also given in 27.

THEOREM 4.1. 27 Let $X$ be a symmetric and positive semidefinite solution to (1.1) such that $A-B B^{*} X$ is stable. Assume that $\left\|X-X_{k}\right\|<1 /\left(3\|B\|^{2}\left\|\Omega_{X}^{-1}\right\|\right)$. If the residual matrix $R_{k}$ satisfies $4\|B\|^{2}\left\|\Omega_{X}^{-1}\right\|^{2}\left\|R_{k}\right\|<1$ then

$$
\left\|X-X_{k}\right\| \leq 2\left\|\Omega_{X}^{-1}\right\|\left\|R_{k}\right\|
$$

We refer the reader to [17 for more refined estimates. This bound is a generalization to the nonlinear case of the well known bound for the (vector) norm of the error when approximately solving a linear system $A x=b$. We note that the "norm of the inverse" 
is replaced here with the norm of the closed-loop operator inverse, which takes into account both the linear and the quadratic coefficient matrices.

We next recall a theorem on the sensitivity of the Lyapunov equation solution.

Theorem 4.2. 24, Theorem 2.2] Let $A$ be stable and let $H$ satisfy $A^{*} H+H A=$ -I. Let $\Delta A$ satisfy $\|\Delta A\|<1 /(2\|H\|)$. Then $A+\Delta A$ is stable.

This result enables us to state that if the error $X-X_{k}$ is small enough, then $X_{k}$ is stabilizable; a similar result can also be found in [27, Lemma 1].

Corollary 4.3. Let $A-B B^{*} X$ be stable and let $X_{k}$ be an approximate solution to (1.1) and $E_{k}=X-X_{k}$. If $\left\|B B^{*} E_{k}\right\|<1 /\left(2\left\|\Omega_{X}^{-1}\right\|\right)$, then $A-B B^{*} X_{k}$ is stable.

Proof. We write $A-B B^{*} X_{k}=\left(A-B B^{*} X\right)+B B^{*} E_{k}=: \widetilde{A}+\Delta \widetilde{A}$. We thus apply Theorem 4.2 to $\widetilde{A}, \Delta \widetilde{A}: \widetilde{A}$ is stable by hypothesis; moreover, if $\left\|B B^{*} E_{k}\right\|=\|\Delta \widetilde{A}\|<$ $1 /\left(2\left\|\Omega_{X}^{-1}\right\|\right)$ then $\widetilde{A}+\Delta \widetilde{A}$ is stable.

Finally, we turn our attention to the special form of the approximate solution, that is $X_{k}=V_{k} Y_{k} V_{k}^{*}$. The following result shows that after $k$ iterations of a projection method, the reduced solution matrix $Y_{k}$ is stabilizing.

Proposition 4.4. Let $T_{k}$ be stable and $\left(T_{k}-B_{k} B_{k}^{*} Y_{k}, C_{k}^{*}\right)$ controllable. Let $Y_{k}$ be the approximation obtained after $k$ iterations of the chosen projection method. Then $T_{k}-B_{k} B_{k}^{*} Y_{k}$ is a stable matrix.

Proof. The symmetric matrix $Y_{k}$ solves the reduced matrix equation $T_{k}^{*} Y+Y T_{k}-$ $Y B_{k} B_{k}^{*} Y+C_{k}^{*} C_{k}=0$. Rewriting the equation, $Y_{k}$ satisfies

$$
\left(T_{k}^{*}-Y_{k} B_{k} B_{k}^{*}\right) Y_{k}+Y_{k}\left(T_{k}-B_{k} B_{k}^{*} Y_{k}\right)+Y_{k} B_{k}^{*} B_{k} Y_{k}+C_{k}^{*} C_{k}=0,
$$

that is, $Y_{k}$ formally solves a Lyapunov equation. Since $Y_{k} B_{k}^{*} B_{k} Y_{k}+C_{k}^{*} C_{k} \geq C_{k}^{*} C_{k}$, Theorem 5.3.2(b) in [29] ensures that the eigenvalues of $T_{k}-B_{k} B_{k}^{*} Y_{k}$ all lie in the open left half-plane, that is the matrix is stable.

Next result tracks the modification in the approximate solution matrix $X_{k}$ as the subspace grows. It is important to realize that in general, the matrices $Y_{k}$ in the sequence are computed by solving a new and expanding Riccati equation, therefore the entries of $Y_{k}$ and $Y_{k+1}$ are not related by a simple explicit recurrence.

Proposition 4.5. Let $X_{j}$ be the approximate solution onto $\mathcal{K}_{j}$ for $j=k, k+1$. Then for $k$ large enough,

$$
\left\|X_{k+1}-X_{k}\right\| \leq 2\left\|\Omega_{Y_{k+1}}^{-1}\right\|\left\|R_{k}\right\| .
$$

Proof. We write $X_{k+1}=V_{k+1} Y_{k+1} V_{k+1}^{*}$ and $X_{k}=V_{k} Y_{k} V_{k}^{*}=V_{k+1} \check{Y}_{k+1} V_{k+1}^{*}$, where $\check{Y}_{k+1}$ is $Y_{k}$ padded with extra rows and columns to match the dimension of $Y_{k+1}$, and we recall that $V_{k+1}=\left[V_{k}, \star\right]$. Moreover, we set $T_{k+1}=\left[T_{k}, t_{k+1}^{(1)} ;\left(t_{k+1}^{(1)}\right)^{\star}, \star\right] . Y_{k+1}$ solves the reduced equation $T_{k+1}^{*} Y+Y T_{k+1}-Y B_{k+1} B_{k+1}^{*} Y+C_{k+1}^{*} C_{k+1}=0$. Substituting instead the matrix $\check{Y}_{k+1}$ we obtain that the residual satisfies

$$
\begin{aligned}
\rho_{k} & :=T_{k+1}^{*} \check{Y}_{k+1}+\check{Y}_{k+1} T_{k+1}-\check{Y}_{k+1} B_{k+1} B_{k+1}^{*} \check{Y}_{k+1}+C_{k}^{*} C_{k} \\
& =V_{k+1}^{*}\left(A^{*} V_{k+1} \check{Y}_{k+1} V_{k+1}^{*}+V_{k+1} \check{Y}_{k+1} V_{k+1}^{*} A-V_{k+1} \breve{Y}_{k+1} V_{k+1}^{*} B B^{*} V_{k+1} \check{Y}_{k+1} V_{k+1}^{*}+C^{*} C\right) V_{k+1} \\
& =V_{k+1}^{*}\left(A^{*} X_{k}+X_{k} A-X_{k} B B^{*} X_{k}+C^{*} C\right) V_{k+1} .
\end{aligned}
$$

Therefore, $\left\|\rho_{k}\right\| \leq\left\|R_{k}\right\|$. Using Theorem 4.1] if $\left\|Y_{k+1}-\check{Y}_{k+1}\right\|<1 /\left(3\left\|B_{k+1}\right\|^{2}\left\|\Omega_{Y_{k+1}}^{-1}\right\|\right)$ and $\left\|\rho_{k}\right\| \leq 1 /\left(4\left\|B_{k+1}\right\|^{2}\left\|\Omega_{Y_{k+1}}^{-1}\right\|^{2}\right)$ then

$$
\left\|Y_{k+1}-\check{Y}_{k+1}\right\| \leq 2\left\|\Omega_{Y_{k+1}}^{-1}\right\|\left\|\rho_{k}\right\| .
$$

Noticing that $\left\|Y_{k+1}-\check{Y}_{k+1}\right\|=\left\|X_{k+1}-X_{k}\right\|$ the result follows. 
5. Rational Krylov subspace approximation. The approximation quality of projection methods depends on the choice of the approximation space $\mathcal{K}_{k}$. In the case of the Lyapunov and Sylvester equations, a classical choice is the Krylov subspace $\mathcal{K}_{k}=$ range $\left(\left[C^{*}, A^{*} C^{*}, \ldots,\left(A^{*}\right)^{k-1} C^{*}\right]\right)$, first introduced for this problem by Saad in 36. Note that in general, $C^{*} \in \mathbb{R}^{n \times p}$ satisfies $p \geq 1$, therefore the space is in fact a "block" space, whose dimension is not greater than $d_{k}=p k$. More recently and motivated by the reduction of dynamical systems, rational Krylov subspaces have shown to be very attractive. For $\mathbf{s}=\left[s_{1}, s_{2}, \ldots\right]$, with $s_{j} \in \mathbb{C}^{+}$, they are given by

$$
\mathcal{K}_{k}\left(A, C^{*}, \mathbf{s}\right):=\operatorname{range}\left(\left[C^{*},\left(A-s_{2} I\right)^{-1} C^{*}, \ldots, \prod_{j=1}^{k-1}\left(A-s_{j+1} I\right)^{-1} C^{*}\right]\right) .
$$

If the problem data are real, the shifts are included in conjugate pairs. Moreover, $\mathfrak{R}\left(s_{j}\right)>0$ therefore all inverses exist for $A$ stable. We remark that the first block of columns generating $\mathcal{K}_{k}$ is simply the matrix $C^{*}$; this corresponds to using an infinite parameter $s_{1}=\infty$ as first shift, and this will be an assumption throughout. Including $C^{*}$ into the space is crucial for convergence, since the whole constant matrix term is exactly represented in the approximation space. The effectiveness of the space now depends on the choice of the parameters $s_{j}, j=2,3, \ldots$. A lot of work has been devoted to the analysis of ideal shifts, due to the relevance of rational Krylov subspaces in eigenproblems 35, 33, matrix function evaluations [22, 14, 23, and Model Order Reduction [21, [34, [15]; we refer the readers to 38] and to the references cited above. We mention that for linear matrix equations, the choice of $s_{j} \in\{0, \infty\}$ seems to be particularly effective in many cases, since the computational cost of solving with the coefficient matrix at each iteration can be somewhat mitigated, without dramatically sacrificing the asymptotic convergence rate. Numerical experiments reported in [40] show that for the algebraic Riccati equation this is no longer the case: the general rational Krylov subspace appears to be superior in all considered examples, in terms of subspace dimension, if the shifts are properly selected. This comparison deserves further study [39]. For the sake of simplicity of exposition or unless it is explicitly stated, in the rest of this section and its subsections we assume that $C$ has a single row, that is $p=1$. There is no relevant difference for $p>1$, except that the same shift is applied to a block of $p$ vectors, and that the involved matrices have dimensions depending on $p k$.

For $k \geq 1$, the rational Krylov subspace with shifts $s_{1}, s_{2}, \ldots, s_{k}$ satisfies the following Arnoldi relation 3 (see, e.g., [12, [30]):

$$
A^{*} V_{k}=V_{k} T_{k}^{*}+\hat{v}_{k+1} g_{k}^{*}, \quad V_{k}^{*} V_{k}=I,
$$

where $\mathcal{K}_{k}=\operatorname{range}\left(V_{k}\right)$, and $\hat{v}_{k+1} \beta=v_{k+1} s_{k}-\left(I-V_{k} V_{k}^{*}\right) A^{*} v_{k+1}$ is the QR decomposition of the right-hand side matrix, and with $g_{k}^{*}=\beta h_{k+1, k} E_{k}^{*} H_{k}^{-1}$. The matrix

$$
\left[\begin{array}{c}
H_{k} \\
h_{k+1, k} E_{k}^{*}
\end{array}\right]
$$

contains the orthogonalization coefficients that generate the orthonormal columns of $V_{k+1}$ (see, e.g., [15]). We set $V_{1} \beta_{0}=C^{*}$, the reduced $\mathrm{QR}$ factorization of $C^{*}$. By

\footnotetext{
${ }^{3}$ The conjugate-transposition in $T_{k}^{*}$ is used for consistency in the notation employed for the reduced Riccati equation.
} 
construction, the matrix $\left[V_{k}, \hat{v}_{k+1}\right]$ has orthonormal columns as well.

Proposition 5.1. The matrix $X_{k}$ satisfies the following algebraic Riccati equation

$$
\left(A^{*}-\hat{v}_{k+1} f_{k}^{*}\right) X+X\left(A-f_{k} \hat{v}_{k+1}^{*}\right)-X B B^{*} X+C^{*} C=0,
$$

where $f_{k}=V_{k} g_{k}$ and $g_{k}$ is as in (5.1).

Proof. The residual satisfies

$$
\begin{aligned}
R_{k} & =\left[V_{k}, \hat{v}_{k+1}\right]\left[\begin{array}{cc}
0 & Y_{k} g_{k} \\
g_{k}^{*} Y_{k} & 0
\end{array}\right]\left[\begin{array}{c}
V_{k}^{*} \\
\hat{v}_{k+1}^{*}
\end{array}\right] \\
& =\hat{v}_{k+1} g_{k}^{*} Y_{k} V_{k}^{*}+V_{k} Y_{k} g_{k} \hat{v}_{k+1}^{*}=\hat{v}_{k+1} g_{k}^{*} V_{k}^{*} X_{k}+X_{k} V_{k} g_{k} \hat{v}_{k+1}^{*} .
\end{aligned}
$$

Substituting into equation (1.2) and collecting terms the result follows.

Since $\left\|f_{k}^{*} X_{k}\right\|=\left\|R_{k}\right\| / \sqrt{2}$, the modified equation of Proposition 5.1 tends to the original Riccati equation as convergence takes place. However, we cannot infer that $X_{k}$ is close to $X$ in the backward error sense, since $\hat{v}_{k+1} f_{k}^{*}$ is not small in general.

5.1. The adaptive rational Krylov subspace. Several different selection strategies have been proposed for the shifts $s_{j}$. In the linear equation case, Penzl (34]) suggested a pre-processing for the computation of a fixed number of shifts, which are then applied cyclically. More recently, a greedy adaptive strategy was proposed in 15. for the same class of problems, which determines the next shift during the computation, so that the process can automatically learn from the convergence behavior of the method. The shifts are selected by minimizing a particular rational function on an approximate and adaptively adjusted spectral region of $A$. In 31 it was observed that for the Riccati equation the inclusion of information on $B B^{*}$ during the shift computation - in the form of eigenvalues of $V_{k}^{*}\left(A^{*}-X_{k} B B^{*}\right) V_{k}$ - may be beneficial in certain cases. In the following we aim to justify this choice. To this end, we need to set a rational function framework that parallels some of the matrix relations obtained in the previous sections.

A relation corresponding to (5.1) can be obtained by using orthogonal rational functions with respect to some inner product; see, e.g., [12. We note that each $v_{j}$ can be written as $v_{j}=\varphi_{j}(A) c /\|c\|$, for some orthogonal rational function $\varphi_{j}=p_{j} / q_{j-1}$, where $p_{j}, q_{j-1}$ are polynomials of degree at most $j$ and $j-1$, respectively. For $j=0$ we define $\varphi_{0}=1$. Let $\Phi_{k-1}(\lambda)=\left[\varphi_{0}(\lambda), \varphi_{1}(\lambda), \ldots, \varphi_{k-1}(\lambda)\right]$. Then,

$$
\lambda \Phi_{k-1}(\lambda)=\Phi_{k-1}(\lambda) T_{k}^{*}+\hat{\varphi}_{k}(\lambda) g_{k}^{*} ;
$$

from (5.3) it follows that $\theta$ is a zero of $\hat{\varphi}_{k}$ if and only $\theta$ is an eigenvalue of $T_{k}$. We refer to [5, section 2.2] for a similar relation, where a different Arnoldi-type relation is used.

A first attempt to justify the use of information from $A-B B^{*} X_{k}$ can be obtained by generalizing the argument in [15, working as if the problem were linear. For the sake of notational simplicity, for the rest of this section we let $\mathcal{A}_{k}=A-B B^{*} X_{k}$ and $\mathcal{T}_{k}=V_{k}^{*} \mathcal{A}_{k} V_{k}=T_{k}-B_{k} B_{k}^{*} Y_{k}$. Using (1.2) we can write the residual as

$$
\begin{aligned}
R_{k} & =\left(A^{*}-X_{k} B B^{*}\right) X_{k}+X_{k}\left(A-B B^{*} X_{k}\right)+C^{*} C+X_{k} B B^{*} X_{k} \\
& =\mathcal{A}_{k}^{*} X_{k}+X_{k} \mathcal{A}_{k}+\mathcal{D}_{k} \mathcal{D}_{k}^{*},
\end{aligned}
$$


where $\mathcal{D}_{k}=\left[C^{*}, X_{k} B\right]$. We observe that all columns of $\mathcal{D}_{k}$ belong to $K_{k}\left(A^{*}, C^{*}, \mathbf{s}\right)$, since $\mathcal{D}_{k}=V_{k}\left[E_{1} \beta_{0}, Y_{k} B_{k}\right]$.

REMARK 5.2. The rational Krylov subspace $K_{k}\left(A^{*}, C^{*}, \mathbf{s}\right)$ satisfies an Arnolditype property for the matrix $\mathcal{A}_{k}$. Indeed,

$$
\begin{aligned}
\mathcal{A}_{k}^{*} V_{k} & =A^{*} V_{k}-X_{k} B B^{*} V_{k} \\
& =V_{k} T_{k}^{*}+\hat{v}_{k+1} g_{k}^{*}-V_{k} Y_{k} B_{k} B_{k}^{*} \\
& =V_{k}\left(T_{k}^{*}-Y_{k} B_{k} B_{k}^{*}\right)+\hat{v}_{k+1} g_{k}^{*}=V_{k} \mathcal{T}_{k}^{*}+\hat{v}_{k+1} g_{k}^{*} .
\end{aligned}
$$

By using the expression of the residual (5.4) as if it were the residual matrix of a Lyapunov equation, we can follow the same reasoning as in [15] for the selection of the next shift. However, as opposed to the linear case, all involved matrices now depend on the iteration $k$. To simplify the presentation, in the following argument we assume that $C^{*}=c \in \mathbb{R}^{n}$. Consider the shifted system $\left(\mathcal{A}_{k}^{*}-s I\right) x=c$, and an approximate solution $x_{k} \in K_{k}\left(\mathcal{A}_{k}^{*}, c, \mathbf{s}\right)$. Then the residual can be written as

$$
c-\left(\mathcal{A}_{k}^{*}-s I\right) V_{k}\left(\mathcal{T}_{k}^{*}-s I\right)^{-1} e_{1} \beta_{0}=\frac{\psi_{k}\left(\mathcal{A}_{k}\right) c}{\psi_{k}(s)}, \quad \psi_{k}(z)=\prod_{j=1}^{k} \frac{z-\lambda_{j}}{z-s_{j}},
$$

where $\lambda_{j}$ are the eigenvalues of $\mathcal{T}_{k}$. The next shift $s_{k+1}$ is then determined so that

$$
s_{k+1}=\arg \left(\max _{s \in \partial \mathbb{S}_{k}}\left|\frac{1}{\psi_{k}(s)}\right|\right),
$$

where $\mathbb{S}_{k} \subset \mathbb{C}^{+}$approximates the mirrored spectral region of $\mathcal{A}_{k}$, and $\partial \mathbb{S}_{k}$ is its border. Note that $\psi_{k}$ is a multiple of $\hat{\varphi}_{k}$ in (5.3). A major practical difference from the adaptive procedure in the Lyapunov equation case is that $\mathbb{S}_{k}$ will change at each iteration in agreement with the modifications in the spectrum of $\mathcal{A}_{k}$. In fact, thanks to the Arnoldi relation of Remark 5.2, the unknown spectral region of $\mathcal{A}_{k}$ is replaced with the spectral region of $\mathcal{T}_{k}$, which is computable after the approximate solution $Y_{k}$ is determined. This approach is precisely the one explored in [31 for the Riccati equation. As opposed to an adaptive shift selection based on $A$ (see, e.g., [40), this approach includes information on the second order coefficient matrix, which may be crucial when the term $-B B^{*} X$ in $A-B B^{*} X$ significantly modifies the spectral properties of $A$ (see Example 5.5). In the next section we give a rigorous formalization of this argument.

5.2. A new expression for the residual and the choice of shifts. In 4 a new expression for the residual of the Sylvester equation was proposed. We extend this expression to the case of the Riccati residual matrix. The new expression allows an interpretation of the two-term sum in (5.2) by means of rational functions. Note that the result also holds for $B=0$, therefore its proof provides a more elementary proof for the Lyapunov equation than in [4].

Proposition 5.3. Assume that the columns of $C^{*}$ belong to Range $\left(V_{k}\right)$, and let $\mathcal{T}_{k}=V_{k}^{*} \mathcal{A}_{k} V_{k}=T_{k}-B_{k} B_{k}^{*} Y_{k}$. Then the residual $R_{k}$ satisfies

$$
R_{k}=\widehat{R}_{k} V_{k}^{*}+V_{k} \widehat{R}_{k}^{*}, \quad \text { with } \quad \widehat{R}_{k}=A^{*} V_{k} Y_{k}+V_{k} Y_{k} \mathcal{T}_{k}+C^{*}\left(C V_{k}\right),
$$

so that $\left\|R_{k}\right\|_{F}=\sqrt{2}\left\|\widehat{R}_{k}\right\|_{F}$. 
Proof. By substituting $\widehat{R}_{k}$ in the expression for $R_{k}$ we obtain,

$$
\begin{aligned}
\widehat{R}_{k} V_{k}^{*}+V_{k} \widehat{R}_{k}^{*}= & A^{*} X_{k}+V_{k} Y_{k} T_{k} V_{k}^{*}-V_{k} Y_{k} B_{k} B_{k}^{*} Y_{k} V_{k}^{*}+C^{*} C \\
& +X_{k} A+V_{k} T_{k}^{*} Y_{k} V_{k}^{*}-V_{k} Y_{k} B_{k} B_{k}^{*} Y_{k} V_{k}^{*}+C^{*} C \\
= & R_{k}+0,
\end{aligned}
$$

where the reduced equation (2.2) and $C^{*} C V V^{*}=C^{*} C$ were used; this proves the first relation. The norm relation follows from $V_{k}^{*} \widehat{R}_{k}=0$, which can be readily verified.

We shall call $\widehat{R}_{k}$ the "semi"-residual matrix. The proposition above shows that the residual norm of the Galerkin method for the Riccati equation is the same as that of an associated Sylvester equation times the constant $\sqrt{2}$. As a consequence, we can at least formally state that $V_{k} Y_{k} V_{k}^{*}$ is a solution to the Riccati equation (1.1), that is $R_{k}=0$, if and only if $Z_{k}=V_{k} Y_{k}$ is the solution to the Sylvester equation

$$
A^{*} Z+Z \mathcal{T}_{k}+C^{*} C V_{k}=0
$$

where $\mathcal{T}_{k}$ typically has dimensions much smaller than $A$. Note that this Sylvester equation is in terms of $A$ (and not of $\mathcal{A}_{k}=A-B B^{*} X_{k}$ ), but also in terms of $\mathcal{T}_{k}$. Let

$$
\psi_{k}(z)=\frac{\operatorname{det}\left(z I-T_{k}\right)}{\prod_{j=1}^{k}\left(z-s_{j}\right)}=\frac{\prod_{j=1}^{k}\left(z-\theta_{j}\right)}{\prod_{j=1}^{k}\left(z-s_{j}\right)},
$$

where $\theta_{j}$ are the eigenvalues of $T_{k}=V_{k}^{*} A V_{k}$. Then the following representation holds for the semi-residual $\widehat{R}_{k}$. The result was first proved for the Sylvester equation in [4] and then generalized to the multi-term linear case in [6]. We prove the result for $C^{*}$ having a single column, the generalization to multiple columns can be obtained by working with each column of $C$, since the whole matrix $C$ is used to build the approximation space.

Theorem 5.4. Assume that $p=1$, that is $C^{*}=c \in \mathbb{R}^{n}$. Let $\psi_{k}$ be the rational function defined in (5.7) and assume that $\mathcal{T}_{k}=V_{k}^{*} \mathcal{A}_{k} V_{k}$ is diagonalizable. The semiresidual $\widehat{R}_{k}$ of Proposition 5.3 satisfies

$$
\widehat{R}_{k}=\psi_{k}\left(A^{*}\right) c c^{*} V_{k}\left(\psi_{k}\left(-\mathcal{T}_{k}\right)\right)^{-1} .
$$

Proof. Let $\mathcal{T}_{k}=Q \Theta Q^{-1}$, with $\Theta=\operatorname{diag}\left(\theta_{1}, \ldots, \theta_{k}\right)$. then the result follows from standard arguments for shifted linear systems. Indeed, substituting this decomposition into $\widehat{R}_{k}$ in Proposition 5.3 it follows that $\widehat{R}_{k} Q=A^{*} V_{k} Y_{k} Q+V_{k} Y_{k} Q \Theta+c c^{*} V_{k} Q$. Let $Z:=V_{k} Y_{k} Q=\left[z_{1}, \ldots, z_{k}\right], c \eta_{j}:=c c^{*} V_{k} Q e_{j}$ and $r_{j}=\widehat{R}_{k} Q e_{j}$, then we have

$$
r_{j}=\left(A^{*}+\theta_{j} I\right) z_{j}+c \eta_{j} .
$$

Due to the Galerkin condition, the residuals $r_{j}$ are all proportional to $\hat{v}_{k+1}$, therefore using (5.7) they can be written as $r_{j}=\hat{\varphi}_{k}\left(A^{*}\right) c \eta_{j} / \hat{\varphi}_{k}\left(-\theta_{j}\right)$. Collecting all columns we get $\widehat{R}_{k} Q=\psi_{k}\left(A^{*}\right) c c^{*} V_{k} Q \psi_{k}(-\Theta)^{-1}$, where we recall that $\psi_{k}$ is a multiple of $\hat{\varphi}_{k}$; multiplying from the right by $Q^{-1}$ the result follows.

We observe that the expression of the semi-residual generalizes the residual formula for the shifted system in (5.5) to the case of matrix equations. The quantity $\left(\psi_{k}\left(-\mathcal{T}_{k}^{*}\right)\right)^{-1}$ plays the same scaling role as the scalar $1 / \psi_{k}(s)$ in the shifted system in (5.5). This new relation thus appears to be of interest on its own. Indeed, while for 
linear matrix equations a parallel with shifted systems had already been performed (see [38, section 4.3] and references therein), the residual matrix associated to the special Sylvester equation (5.6) had not been explicitly written down in terms of polynomials or rational functions.

The new expression for $\widehat{R}_{k}$ suggests a way to determine the next shift $s_{k+1}$. Indeed, we first recall that the numerator of the rational function $\psi_{k}$ is the characteristic polynomial of $T_{k}$, which thus minimizes the numerator of $\psi_{k}$ among all monic polynomials of degree $k$. This makes $\left\|\psi_{k}(A) c\right\|$ small among all rational functions $\psi_{k}$ with fixed denominator and monic numerator. With the next shift we thus want to make the quantity $\left(\psi_{k}\left(-\mathcal{T}_{k}\right)\right)^{-1}$ smaller in the expression for $\widehat{R}_{k}$. Therefore, we determine for which $z$ in the spectral region of $\mathcal{T}_{k}$ the quantity $\left(\psi_{k}(-z)\right)^{-1}$ is large, and add a root there for the construction of the next function $\varphi_{k}$. Therefore, $s_{k+1}$ is chosen as the solution to the following problem

$$
s_{k+1}=\arg \max _{s \in \partial \mathbb{S}_{k}}\left|\frac{1}{\psi_{k}(s)}\right|=\arg \max _{s \in \partial \mathbb{S}_{k}}\left|\frac{\prod_{j=1}^{k}\left(s-s_{j}\right)}{\operatorname{det}\left(s I-\mathcal{T}_{k}\right)}\right|,
$$

where here $\mathbb{S}_{k}$ is a region enclosing the eigenvalues of $-\mathcal{T}_{k}$ and $\partial \mathbb{S}_{k}$ is its border. This approach should be compared with the original algorithm that uses $T_{k}$ instead. This modified selection strategy can be implemented very easily, with a slight modification of the original algorithm in [15]: the algorithm needs to compute the eigenvalues of $\mathcal{T}_{k}=T_{k}-B_{k} B_{k}^{*} Y_{k}$ instead of those of $T_{k}$ to determine the corresponding convex hull. It is interesting to observe that for $A$ Hermitian, working with the non-Hermitian matrix $\mathcal{T}_{k}$ appears to be more complex than working with the Hermitian matrix $T_{k}$. On the other hand, the matrix $\mathcal{T}_{k}$ has a key role in the Riccati semi-residual matrix, and it takes into account the nonlinear term in the original equation. Clearly, if the convex hulls of $T_{k}$ and $\mathcal{T}_{k}$ are similar, and the same for those of $A$ and of $A-B B^{*} X$, then no major differences will be observed between the two selection strategies. In other words, if the field of values are similar, then the projection method based only on the linear part will be able to decrease $\left\|\widehat{R}_{k}\right\|$ with a similar convergence rate.

We next report an example illustrating the expected behavior of the rational Krylov method with or without the inclusion of the term $-B_{k} B_{k}^{*} Y_{k}$ in the computation of the spectral region in 5.8 .

EXAMPLE 5.5. We consider a small built-up example, where $A$ is the Toeplitz matrix $A=-$ toeplitz $(-1,-1.5,2.8,1,1,1)$ of size $n=700$ (this small size allows us to easily compute all quantities for this theoretical analysis). Moreover, $B=t \mathbf{1}$ and $C=[1,-2,1,-2,1,-2, \ldots]$; this example is motivated by an example with similar data in [31]. The parameter $t$ takes the values $t_{j}=5 \cdot 10^{-j}$, so that for $j=3,\|B\| \approx 1$. The left plot of Figure 5.1 shows the convergence history (relative residual norm) of the rational Krylov method for each of the three different values of $t$, when the shifts are adaptively computed on the spectral region of $\mathcal{T}_{k}$, as in (5.8). The right plot of Figure 5.1 shows the modification of the convex hull of $A^{*}-X B B^{*}$ as $t$ varies. For the larger values of $t_{j}$, the magnitude of $B$ significantly influences the spectral convex hull; by using the modified shift computation strategy, the method is able to adapt to this change and capture the new problem features. We remark that by using spectral information of $T_{k}$ instead, the method takes about 12 iterations to converge, irrespective of the value of $t$. We notice that for $B$ of rank one, the matrix $X B B^{*}$

\footnotetext{
${ }^{4}$ The Matlab (32]) code for the rational Krylov subspace method for the Riccati equation is available at the author's webpage http://www.dm.unibo.it/ simoncin/software.html
} 
is also rank one, with a real positive eigenvalue whose magnitude depends on $B$ and thus on $t$. For $\|B\|$ large, Figure 5.1 shows that for this example only one eigenvalue of $A^{*}$ is significantly perturbed in $A^{*}-X B B^{*}$, causing the extension of the original spectrum to the left, by an amount depending on $t$.
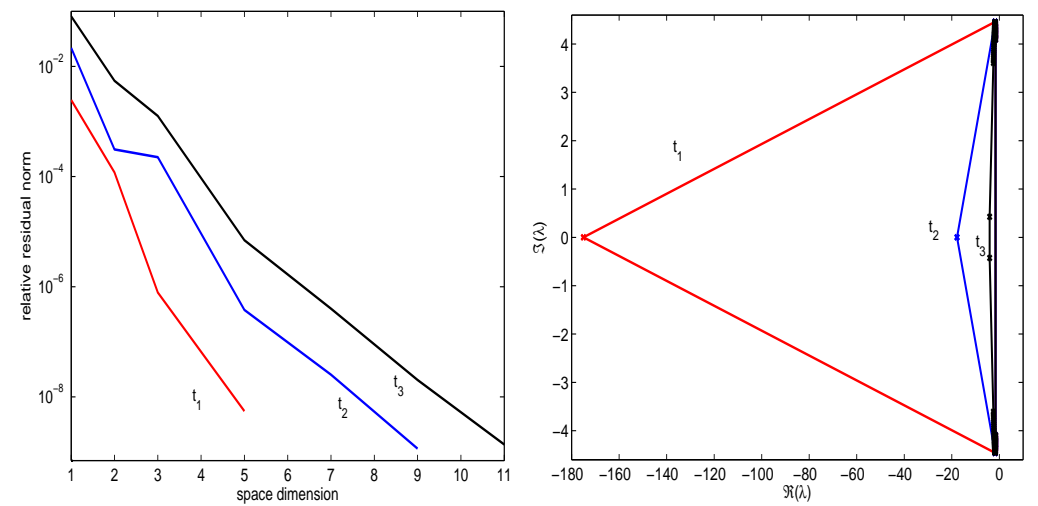

FIG. 5.1. Example 5.5. Left: Convergence history of rational Krylov method with modified shift selection as $t$ varies. Right: convex hull of $A^{*}-X B B^{*}$ as $t$ varies.

Example 5.5 shows that for these data, the magnitude of $B$ influences the residual convergence of the modified method in a counterintuitive way: the larger its norm, the faster the method convergence. By using the modified shift selection, the isolated eigenvalue of $A^{*}-X B B^{*}$ (see Figure 5.1) is readily located, and the residual is forced to be small in that region as well.

In the next example we explore the influence of the nonsymmetry of $\mathcal{T}_{k}$ in the shift computation, when $A$ is symmetric.

EXAMPLE 5.6. We consider the same setting as for Example 5.5, except that now $A=A_{0} \otimes I_{n_{0}}+I_{n_{0}} \otimes A_{0}$, with $A_{0}=$ toeplitz $(1, \underline{-2}, 1) \in \mathbb{R}^{n_{0} \times n_{0}}$, with $n_{0}=30$ and $\otimes$ the Kronecker product, giving rise to a $900 \times 900$ symmetric negative definite matrix. These data represent the scaled finite difference discretization of the Laplacian on the unit square with homogeneous boundary conditions. As $t$ varies, we compare the performance of the method when $\mathbb{S} \subset \mathbb{R}$ is associated with the symmetric matrix $T_{k}$, with the case when $\mathbb{S} \subset \mathbb{C}$ due to the use of $\mathcal{T}_{k}$; to emphasize this dependence will shall use $\mathbb{S}\left(\mathrm{T}_{k}\right)$ and $\mathbb{S}\left(\mathcal{T}_{k}\right)$, respectively. Table 5.1 shows the space dimension required by the two approaches to reach an absolute residual norm of $10^{-9}$. Shown are also the absolute residual and error norms at convergence, and the norm of the exact solution. We report that all computed shifts were real also for $\mathcal{T}_{k}$. The table shows that the number of iterations for the residual to converge is always smaller when $\mathbb{S}\left(\mathcal{T}_{k}\right)$ is used, and it decreases with the magnitude growth of the $B$ term, as in the previous example. We also notice that when using $T_{k}$, the final error is significantly smaller than in the modified version of the method; apparently, the residual lags behind in convergence, when $\mathbb{S}\left(T_{k}\right)$ is used.

Figure 5.2 displays the residual convergence history for the two approaches, as $t$ varies. The initial steep phase of the residual in the modified approach is granted by the fact that the approximation space immediately locates the isolated eigenvalue, and that the residual appears to have a large component in the corresponding eigendirection. After that, the convergence behavior depends on the rest of the spectrum. The original solver maintains the same convergence rate for all values of $t$. 


\begin{tabular}{|llrccc|}
\hline$t$ & $\begin{array}{l}\text { Spectral } \\
\text { Region }\end{array}$ & $\begin{array}{r}\text { Space } \\
\text { dim. }\end{array}$ & $\left\|R_{k}\right\|_{F}$ & $\left\|X-X_{k}\right\|_{F}$ & $\|X\|_{F}$ \\
\hline $10^{3}$ & $\mathbb{S}\left(\mathrm{T}_{k}\right)$ & 21 & $1.8500 \mathrm{e}-10$ & $1.6646 \mathrm{e}-13$ & $4.9999 \mathrm{e}-03$ \\
& $\mathbb{S}\left(\mathcal{T}_{k}\right)$ & 3 & $8.5599 \mathrm{e}-10$ & $1.4389 \mathrm{e}-10$ & \\
\hline $10^{2}$ & $\mathbb{S}\left(\mathrm{T}_{k}\right)$ & 23 & $3.1915 \mathrm{e}-10$ & $3.0155 \mathrm{e}-13$ & $4.9994 \mathrm{e}-02$ \\
& $\mathbb{S}\left(\mathcal{T}_{k}\right)$ & 7 & $4.9612 \mathrm{e}-10$ & $1.0148 \mathrm{e}-10$ & \\
\hline 10 & $\mathbb{S}\left(\mathrm{T}_{k}\right)$ & 25 & $9.6706 \mathrm{e}-10$ & $2.5302 \mathrm{e}-13$ & $4.9938 \mathrm{e}-01$ \\
& $\mathbb{S}\left(\mathcal{T}_{k}\right)$ & 9 & $9.0853 \mathrm{e}-10$ & $2.2998 \mathrm{e}-10$ & \\
\hline
\end{tabular}

Example [5.6] Comparison of performance for A symmetric. Number of iterations for the two variants for the relative residual norm and final accuracies to go below $10^{-9}$.

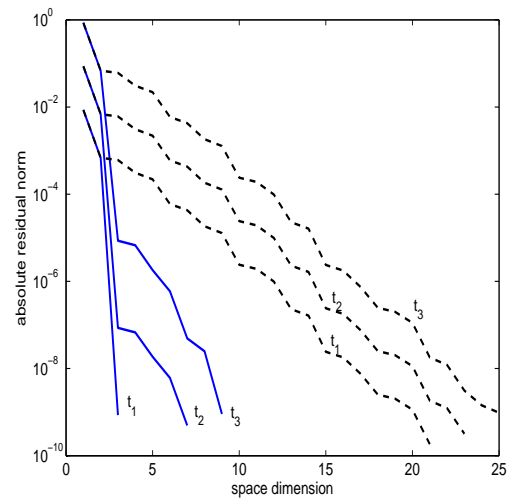

FIG. 5.2. Example 5.6. Convergence history of rational Krylov method with and without modified shift selection as $t$ varies. Solid curves: use of $\mathcal{T}_{k}$. Dashed curves: use of $T_{k}$.

By generalizing field of values results in [13, 4] it may be possible to exploit the semi-residual form to analyze the convergence of the method, and its dependence on $\mathcal{T}_{k}$. A shortcoming in the analysis is that the field of values of the non-Hermitian matrix $\mathcal{T}_{k}$ depends on $k$, and that its relation with the field of values of $A^{*}-X B B^{*}$ is not easy to formalize, especially at an early stage of the convergence history. Resorting to the residual expression in (5.4), it is possible to exploit some of the results available in the literature for the Lyapunov equation. For instance, if the field of values of $A^{*}-X B B^{*}$ and of $A^{*}-X_{k} B B^{*}$ is contained in a disk of center $c>0$ and radius equal to one for all $k$, then using [13. Theorem 4.11] we can state that the error satisfies

$$
\varlimsup_{k \rightarrow \infty}\left\|X-X_{k}\right\|^{\frac{1}{k}} \leq \frac{2 c^{2}+c-1-(2 c+1) \sqrt{c^{2}-1}}{c+1+\sqrt{c^{2}-1}}=: \gamma .
$$

The following example shows that this asymptotic bound can be descriptive of the actual behavior.

EXAMPLE 5.7. We consider $A=-1 /(3.2) A_{0}-I$ where $A_{0}$ is the Grcar matrix, $A_{0}=$ toeplitz $(-1, \underline{1}, 1,1,1) \in \mathbb{R}^{n \times n}, n=1600, C=\mathbf{1} /\|\mathbf{1}\|$ and $B \in \mathbb{R}^{n \times p}, p=20$ with normally distributed random numbers, normalized so that its norm is about $5 \cdot 10^{-2}$. The left plot of Figure 5.3 shows the computed spectrum of $A$ (' $x$ ' symbol), that of $A^{*}-X B B^{*}$ ("o'symbol), the border of the field of values of both $A$ and $A^{*}-X B B^{*}$ (thin line), and the circle of center $c=1.25$ and radius one, enclosing the field of values. The right plot of Figure 5.3 displays the error norm history of the modified method 
(dashed line), and $10^{-2} \gamma^{k}$, The convergence rate is well captured by the theoretical estimate $\gamma$ at the early stage of the iterations.
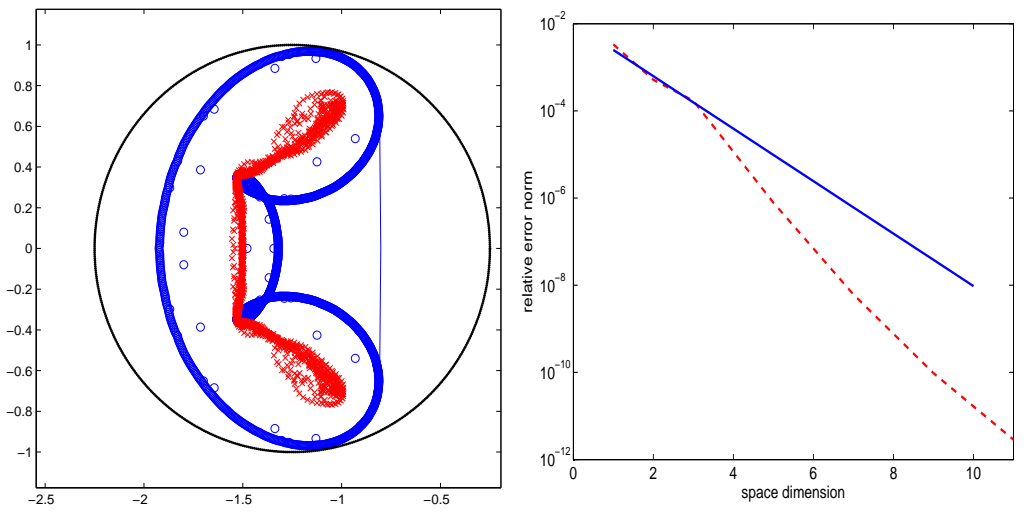

FIG. 5.3. Example 5.7. Left: Field of values and eigenvalue location. Right: Error norm convergence history and estimate $\gamma^{k}$.

6. Approximation of an invariant subspace. In this section we discuss the natural, albeit gone almost unnoticed, role of the approximation matrix $X_{k}$ in the eigenvalue context. The problem of solving the large scale algebraic Riccati equation for $X \geq 0$ can be transformed into the problem of computing an approximate basis for the stable invariant subspace of the following Hamiltonian matrix (see, e.g., [29])

$$
\mathcal{H}=\left[\begin{array}{cc}
A & -B B^{*} \\
-C^{*} C & -A^{*}
\end{array}\right] .
$$

Several different approaches have been devised to this end, see, e.g., [1, [7, 31] and references therein. Here we show that the projection process described in the previous sections can be equivalently applied to this context, providing further motivation for the method.

Let $X_{k}$ be the approximate solution to (1.1) obtained by the rational Krylov subspace method. For some $L \in \mathbb{R}^{n \times n}$ consider the eigenvalue residual

$$
\mathcal{S}_{k}=\left[\begin{array}{cc}
A & -B B^{*} \\
-C^{*} C & -A^{*}
\end{array}\right]\left[\begin{array}{c}
I \\
X_{k}
\end{array}\right]-\left[\begin{array}{c}
I \\
X_{k}
\end{array}\right] L .
$$

For $L=A-B^{*} B X_{k}$, the invariant space residual $\mathcal{S}_{k}$ and the matrix equation residual $R_{k}$ can be easily related, since

$$
\mathcal{S}_{k}=\left[\begin{array}{cc}
A & -B B^{*} \\
-C^{*} C & -A^{*}
\end{array}\right]\left[\begin{array}{c}
I \\
X_{k}
\end{array}\right]-\left[\begin{array}{c}
I \\
X_{k}
\end{array}\right]\left(A-B^{*} B X_{k}\right)=\left[\begin{array}{c}
0 \\
R_{k}
\end{array}\right],
$$

so that $\left\|\mathcal{S}_{k}\right\|=\left\|R_{k}\right\|$. As a consequence of Proposition 5.1 the following result holds.

Proposition 6.1. The columns of the matrix $\left[I ; X_{k}\right]$ span an invariant subspace of the matrix

$$
\mathcal{H}_{k}=\left[\begin{array}{cc}
A-f_{k} \hat{v}_{k+1}^{*} & -B B^{*} \\
-C^{*} C & -\left(A-f_{k} \hat{v}_{k+1}^{*}\right)^{*}
\end{array}\right],
$$

and the spectrum of $T_{k}^{*}-Y_{k} B_{k} B_{k}^{*}$ is a subset of the spectrum of $A^{*}-X_{k} B B^{*}-\hat{v}_{k+1} f_{k}^{*}$. 
Proof. Writing the eigenresidual

$$
\mathcal{S}_{k}=\left[\begin{array}{cc}
A-f_{k} \hat{v}_{k+1}^{*} & -B B^{*} \\
-C^{*} C & -\left(A-f_{k} \hat{v}_{k+1}^{*}\right)^{*}
\end{array}\right]\left[\begin{array}{c}
I \\
X_{k}
\end{array}\right]-\left[\begin{array}{c}
I \\
X_{k}
\end{array}\right]\left(A-f_{k} \hat{v}_{k+1}^{*}-B^{*} B X_{k}\right)
$$

and using Proposition 5.1 we readily see that $\mathcal{S}_{k}=0$.

To prove the second assertion, we use the Arnoldi relation in (5.1). Let $(\theta, z)$ be an eigenpair of $T^{*}-Y_{k} B_{k} B_{k}^{*}$. Then

$$
\begin{aligned}
\left(A^{*}-\hat{v}_{k+1} f_{k}^{*}-X_{k} B B^{*}\right) V_{k} z & =\left(A^{*} V_{k}-\hat{v}_{k+1} f_{k}^{*} V_{k}-X_{k} B B^{*} V_{k}\right) z \\
& =\left(V_{k} T_{k}^{*}+\hat{v}_{k+1} g_{k}^{*}-\hat{v}_{k+1} f_{k}^{*} V_{k}-X_{k} B B^{*} V_{k}\right) z \\
& =\left(V_{k} T_{k}^{*}-X_{k} B B^{*} V_{k}\right) z=V_{k}\left(T_{k}^{*}-Y_{k} B_{k} B_{k}^{*}\right) z=V_{k} z \theta,
\end{aligned}
$$

and the result follows.

The result above states that the approximate Riccati solution is associated with an invariant subspace of a modification of the original matrix in (6.1), and that the spectrum of $T_{k}^{*}-Y_{k} B_{k} B_{k}^{*}$ is a portion of the spectrum of this modified problem. These properties are a consequence of the Arnoldi relation (5.1), which indeed states that $V_{k}$ is an invariant subspace basis of a modification of $A^{*}$, namely of $A^{*}-\hat{v}_{k+1} f_{k}^{*}$. What is noticeable in our context is that we can relate the spectral region over which we seek the next shift in (5.8) with the spectral region of a relevant matrix back in $\mathbb{R}^{n}$.

The approximation process leading to the computation of $Y_{k}$ can be interpreted as a Galerkin method for the eigenvalue problem associated with $\mathcal{H}$. Consider the space

$$
\mathbb{V}_{k}=\operatorname{range}\left(\left[\begin{array}{cc}
V_{k} & 0 \\
0 & V_{k}
\end{array}\right]\right)=: \operatorname{range}\left(\mathcal{V}_{k}\right)
$$

Then by projecting $\mathcal{H}$ onto the space we obtain,

$$
\mathcal{V}_{k}^{*} \mathcal{H} \mathcal{V}_{k}=\left[\begin{array}{cc}
V_{k}^{*} A V_{k} & -V_{k}^{*} B B^{*} V_{k} \\
-V^{*} C^{*} C V_{k} & -V_{k}^{*} A^{*} V_{k}
\end{array}\right]=\left[\begin{array}{cc}
T_{k} & -B_{k} B_{k}^{*} \\
-C_{k}^{*} C_{k} & -T_{k}^{*}
\end{array}\right] .
$$

The block matrix on the right-hand side is the Hamiltonian matrix associated with the reduced system. Using the reduced Riccati equation, it holds that

$$
\left[\begin{array}{cc}
T_{k} & -B_{k} B_{k}^{*} \\
-C_{k}^{*} C_{k} & -T_{k}^{*}
\end{array}\right]\left[\begin{array}{c}
I \\
Y_{k}
\end{array}\right]=\left[\begin{array}{c}
I \\
Y_{k}
\end{array}\right]\left(T_{k}-B_{k} B_{k}^{*} Y_{k}\right),
$$

with $Y_{k}$ stabilizing. In terms of original space dimensions, let $\mathcal{V}_{k}\left[I ; Y_{k}\right]=\left[V_{k} ; V_{k} Y_{k}\right]$ be the computed approximate eigenbasis. Then the residual is given by

$$
\mathcal{S}_{k}=\left[\begin{array}{cc}
A & -B B^{*} \\
-C^{*} C & -A^{*}
\end{array}\right]\left[\begin{array}{c}
V_{k} \\
V_{k} Y_{k}
\end{array}\right]-\left[\begin{array}{c}
V_{k} \\
V_{k} Y_{k}
\end{array}\right]\left(T_{k}-B_{k} B_{k}^{*} Y_{k}\right) .
$$

It readily follows that the eigenresidual is orthogonal to the generated space, that is it holds that $\left(\mathcal{V}_{k}\right)^{*} \mathcal{S}_{k}=0$, therefore it satisfies a standard Galerkin condition. As a consequence, for $T_{k}-B_{k} B_{k}^{*} Y_{k}$ stable, $\mathcal{V}_{k}\left[I ; Y_{k}\right]$ approximates a basis of a stable invariant subspace of the matrix $\mathcal{H}$ in the sense of Galerkin projection methods. 
7. Conclusions. By looking at the problem from different but highly related perspectives, we have shown that projection methods are a natural device for solving the algebraic Riccati equation. In particular, the reduced equation solves a reduced linear-quadratic optimization problem, as is typical of model order reduction techniques. By using classical arguments, we have related the residual with the error of the current approximation. Moreover, we have derived a new expression for the residual in terms of rational functions; this expression allows us to justify recent algorithmic strategies for the choice of the shift parameters used in the construction of the approximation space. In addition, this expression highlights the role of the quadratic term, and explains why it often happens that good convergence occurs even without taking the quadratic term into account during the construction of the approximation space. The new relations for the residual in terms of rational functions can be the starting point for a convergence analysis of the method. We notice that while we have focussed on generic rational Krylov subspaces in section [5, many of the stated results hold for other choices of approximation spaces, and in particular for polynomial and extended Krylov subspaces.

Finally, we have shown that the computed quantities correspond to a Galerkin approximation of the eigenvalue problem associated with the Hamiltonian matrix of the dynamical system.

Acknowledgements. We would like to thank Dario Bini and Daniel Szyld for insightful comments. This research is supported in part by the FARB12SIMO grant of the Università di Bologna, and by INdAM-GNCS under the 2016 Project Equazioni e funzioni di matrici con struttura: analisi e algoritmi.

\section{REFERENCES}

[1] L. Amodei And J.-M. Buchot, An invariant subspace method for large-scale algebraic Riccati equation, Applied Numerical Mathematics, 60 (2010), pp. 1067-1082.

[2] A. Antoulas, C. Beattie, and S. Gugercin, Interpolatory model reduction of large-scale dynamical systems, in Efficient Modeling and Control of Large-Scale Systems, J. Mohammadpour and K. Grigoriadis, eds., Springer-Verlag, February 2010. ISBN 978-1-4419-5756-6.

[3] A. C. Antoulas, Approximation of large-scale Dynamical Systems, Advances in Design and Control, SIAM, Philadelphia, 2005.

[4] B. Beckermann, An Error Analysis for Rational Galerkin Projection applied to the Sylvester Equation, SIAM J. Numer. Anal., 49 (2011), pp. 2430-2450.

[5] B. Beckermann, S. Güttel, and R. Vandebril, On the convergence of rational ritz values, SIAM J. Matrix Anal. Appl., 31 (2010), pp. 1740-1774.

[6] B. Beckermann, D. Kressner, and C. Tobler, An error analysis of Galerkin projection methods for linear systems with tensor product structure, SIAM J. Numer. Anal., 51 (2013), pp. 3307-3326.

[7] P. Benner and Z. Bujanovic, On the solution of large-scale algebraic Riccati equations by using low-dimensional invariant subspaces, Linear Algebra and Its Applications, 488 (2016), pp. $430-459$.

[8] P. Benner, J.-R. Li, And T. Penzl, Numerical solution of large-scale Lyapunov equations, Riccati equations, and linear-quadratic optimal control problems, Num. Lin. Alg. with Appl., 15 (2008), pp. 1-23.

[9] P. Benner, V. Mehrmann, And D. S. (eds), Dimension Reduction of Large-Scale Systems, Lecture Notes in Computational Science and Engineering, Springer-Verlag, Berlin/Heidelberg, 2005.

[10] P. Benner AND J. SAAK, A Galerkin-Newton-ADI method for solving large-scale algebraic Riccati equations, Tech. Rep. SPP1253-090, Deutsche Forschungsgemeinschaft - Priority Program 1253, 2010.

[11] D. Bini, B. Iannazzo, and B. Meini, Numerical Solution of Algebraic Riccati Equations, SIAM, Philadelphia, 2012. 
[12] K. Deckers And A. Bultheel, Rational Krylov sequences and orthogonal rational functions, tech. rep., Department of Computer Science, K.U.Leuven, 2007.

[13] V. Druskin, L. Knizhnerman, And V. Simoncini, Analysis of the rational Krylov subspace and ADI methods for solving the Lyapunov equation, SIAM J. Numer. Anal., 49 (2011), pp. $1875-1898$.

[14] V. Druskin, C. Lieberman, And M. Zaslavsky, On adaptive choice of shifts in rational Krylov subspace reduction of evolutionary problems, SIAM J. Sci. Comput., 32 (2010), pp. 2485-2496.

[15] V. Druskin and V. Simoncini, Adaptive rational Krylov subspaces for large-scale dynamical systems, Systems and Control Letters, 60 (2011), pp. 546-560.

[16] F. Feitzinger, T. Hylla, and E. W. Sachs, Inexact Kleinman-Newton method for Riccati equations, SIAM J. Matrix Anal. Appl., 31 (2009), pp. 272-288.

[17] P. Gahinet And A. J. Laub, Computable bounds for the sensitivity of the algebraic Riccati equation, SIAM J. Control and Opt., 28 (1990), pp. 1461-1480.

[18] K. Gallivan, A. Vandendorpe, And P. V. Dooren, Sylvester equations and projection-based model reduction, J. Comput. Appl. Math., 162 (2004), pp. 213-229.

[19] L. GRASEDYCK, Nonlinear multigrid for the solution of large-scale Riccati equations in low-rank and $\mathcal{H}$-matrix format, Numer. Linear Algebra Appl., 15 (2008), pp. 779-807.

[20] L. Grasedyck, W. Hackbusch, and B. KhoromskiJ, Solution of large scale algebraic matrix Riccati equations by use of hierarchical matrices, Computing, 70 (2003), pp. 121-165.

[21] E. GRImme, Krylov projection methods for model reduction, PhD thesis, The University of Illinois at Urbana-Champaign, 1997.

[22] S. GütTEL, Rational Krylov approximation of matrix functions: Numerical methods and optimal pole selection, GAMM Mitteilungen, 36 (2013), pp. 8-31.

[23] S. Güttel and L. Knizhnerman, Automated parameter selection for rational Arnoldi approximation of Markov functions, Proc. Appl. Math. Mech., 11 (2011), pp. 15-18.

[24] G. Hewer And C. Kenney, The sensitivity of the stable Lyapunov equation, SIAM J. Control and Optimization, 26 (1988), pp. 321-344.

[25] M. Heyouni And K. JBilou, An extended Block Krylov method for large-scale continuous-time algebraic Riccati equations, ETNA, 33 (2008-2009), pp. 53-62.

[26] K. JBILOU, Block Krylov subspace methods for large algebraic Riccati equations, Numerical Algorithms, 34 (2003), pp. 339-353.

[27] C. Kenney, A. J. Laub, and M. Wette, Error bounds for Newton refinement of solutions to algebraic Riccati equations, Math. Control Signals Systems, 3 (1990), pp. 211-224.

[28] D. L. Kleinman, On an Iterative Technique for Riccati Equation Computations, IEEE Transactions on Automatic Control, 13 (1968), pp. 114-115.

[29] P. Lancaster and L. Rodman, Algebraic Riccati equations, Oxford Univ. Press, 1995.

[30] Y. Lin And V. Simoncini, Minimal residual methods for large scale Lyapunov equations, Applied Num. Math., 72 (2013), pp. 52-71.

[31] Y. Lin And V. Simoncini, A new subspace iteration method for the algebraic Riccati equation, Numerical Linear Algebra w/Appl., 22 (2015), pp. 26-47.

[32] The MathWorks, Inc., MATLAB 7, r2013b ed., 2013.

[33] K. H. A. Olsson And A. RuHe, Rational Krylov for eigenvalue computation and model order reduction, BIT Numerical Mathematics, 46 (2006), pp. 99-111.

[34] T. PEnzL, A cyclic low-rank Smith method for large sparse Lyapunov equations, SIAM J. Sci. Comput., 21 (2000), pp. 1401-1418.

[35] A. RuHe, Rational Krylov sequence methods for eigenvalue computation, Lin. Alg. Appl., 58 (1984), pp. 391-405.

[36] Y. SAAD, Numerical solution of large Lyapunov equations, in Signal Processing, Scattering, Operator Theory, and Numerical Methods. Proceedings of the international symposium MTNS-89, vol III, M. A. Kaashoek, J. H. van Schuppen, and A. C. Ran, eds., Boston, 1990, Birkhauser, pp. 503-511.

[37] W. H. A. Schilders, H. A. van der Vorst, And J. Rommes, Model Order Reduction: Theory, Research Aspects and Applications, Springer-Verlag, Berlin/Heidelberg, 2008.

[38] V. Simoncini, Computational methods for linear matrix equations, tech. rep., Alma Mater Studiorum - Università di Bologna, 2013. SIAM Review, Sept 2016.

[39] - On the extended Krylov subspace method for the algebraic Riccati equation, January 2016. In preparation.

[40] V. Simoncini, D. B. Szyld, And M. Monsalve, On two numerical methods for the solution of large-scale algebraic Riccati equations, IMA Journal of Numerical Analysis, 34 (2014), pp. 904-920.

[41] G. W. Stewart and J.-G. Sun, Matrix Perturbation Theory, Academic Press, 1990. 